\title{
Wideband Focused Transducer Array for Optoacoustic Tomography
}

\author{
Varvara A. SIMONOVA ${ }^{1}$, Alexander A. KARABUTOV ${ }^{2}$, Tatyana D. KHOKHLOVA \\ ${ }^{1}$ Joint Institute on Laser and Information Technologies, Russian Academy of Science, 1 Svyatoozerskaya \\ St., 140700 Shatura, Moscow Region, Russian Federation, \\ e-mail: simvar@mail.ru \\ ${ }^{2}$ Moscow State University International Laser Centre, Leninskie gory, 1, 119992 Moscow, Russian \\ Federation
}

\begin{abstract}
:
In this work, a multi-element transducer has been developed for the optoacoustic tomography of biological objects with a given spatial resolution in three dimensions (axial resolution in the image plane was $\Delta \mathrm{x}=0.1 \mathrm{~mm}$, lateral resolution in the image plane was $\Delta \mathrm{z}=1.6 \mathrm{~mm}$, lateral resolution in the plane perpendicular to the image plane was $\Delta y=0.5 \mathrm{~mm}$ ). The transducer consists of a set of 8 linear PVDF piezoelectric films (width is $2 \mathrm{~mm}$ ) located in a plane with 1-mm gap and focused by a cylindrical acoustic lens.

Map of the transducer focal area has been experimentally investigated. The resolution $\Delta y$ was obtained from the map and is equal to $0.5 \mathrm{~mm}$. Image of a focused point source has been rendered based on experimentally obtained optoacoustic signals. The $\mathrm{x}$-axis resolution and the $\mathrm{z}$-axis resolution were calculated from the focused point source image reconstructed using a back-projection algorithm and are equal to $0.1 \mathrm{~mm}$ and $2 \mathrm{~mm}$ respectively.
\end{abstract}

Keywords: optoacoustic tomography, transducer array, breast cancer

\section{Introduction}

Laser optoacoustic (OA) tomography is new promising technique for biodiagnostics. Applications of the OA method differ on the required spatial resolution defined by frequency bandwidth [1]. Early stage detection of breast cancer now seems to be one of the most important applications of the OA method. Such diagnostics require visualization of a small (lateral dimension is a few millimeters) light absorbing object located several centimeters deep into a light scattering medium.

For in-vivo diagnostics, two-dimensional images are preferable since data can be gathered and processed in real time [2]. A 2D image corresponds to the section of the heat sources distribution in the image plane. The section thickness corresponds to the resolution in the direction perpendicular to the image plane and is defined by the design of the transducer array. Low resolution in that direction was the main reason of the decreased quality in the design of all transducer arrays described before [3]. This problem can be solved by using an array of focused transducers $[4,5,6]$. The focused transducer sensitivity is distributed in a narrow focal region. Consequently, the sensitivity of the entire antenna is located in the image plane [7].

The shape of the surface where the transducers are located is a key parameter defining the type of focusing of the multi-element antenna. Generally the shape of the surface is not only defined by the required image resolution but also by the application for which 
the antenna will be used. For the application under consideration (early breast cancer detection) we will consider cylindrical shape of the antenna surface. However, from the technical standpoint, it is better using plane transducers with a focusing acoustic lens [8]. It was shown [3, 9] that signal-to-noise ratio and sensitivity increase for such system leading to an improvement of the image contrast.

Currently there is no analytical algorithms for the calculation of multi-element focused antenna parameters. In ref. [10], the relationship between the resolution and the flare angle of an antenna was numerically calculated and experimentally confirmed for the case of spherically focused radiator of continuous ultrasound waves. However, the relationship between the parameters of the antenna and the required resolution should be known to obtain an antenna with the given characteristics of reconstructed image. In this paper, we propose an analytical algorithm which allows one to calculate the characteristics and the geometric parameters of wideband multi-element antenna with a high spatial resolution for OA tomography.

\section{Theoretical model}

The focused transducers located on the concave plane perpendicular to the axis of cylinder damping surface are considered. Then the plane transducers located on focusing acoustic lens (Fig. 1) are investigated.

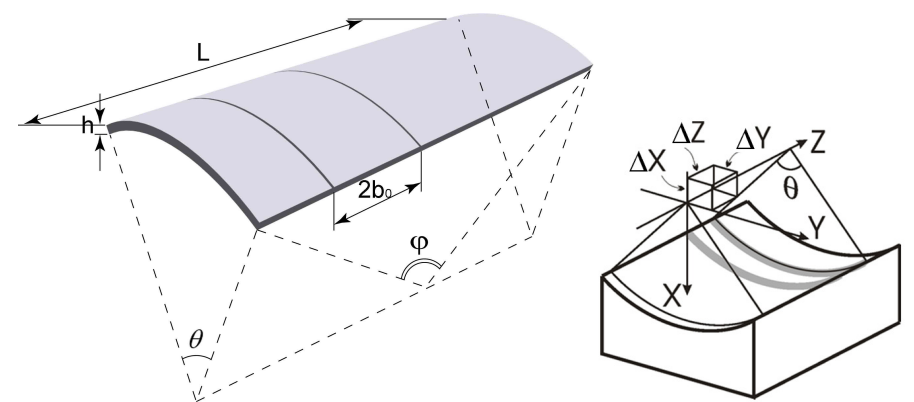

Figure 1. Geometry of considered antenna and resolutions of image reconstructed by means of the antenna; $2 b_{0}$ - piezoelectric element width; $h$ - piezoelectric element thickness; $L$ - antenna length; $\varphi, \theta$

- flare angles of the antenna in perpendicular planes.

The input data for the calculation of the antenna design parameters are the voxel dimensions $\Delta x, \Delta y, \Delta z$ (Fig. 1). The required voxel dimensions are defined by a specified application of OA tomography.

The electric signal of a transducer corresponds to the convolution of the input acoustic signal and the transducer transient response [5, 11]. The spectral sensitivity of the transducer defines the bandwidth of the received signal which consequently defines the value of the longitudinal resolution $\Delta x$. Besides, the frequency of the main transducer resonance must be at least greater than the upper boundary frequency $f_{\max }$ in the spectrum of the received wideband optoacoustic signal in order to eliminate visible distortions. Such a limitation defines the maximum thickness of the transducer. 
The equation of diffraction can be solved analytically for the case of monochromatic waves [12]. However OA signals are characterized by wideband spectra [5]. Therefore, a higher spatial resolution requires a wider frequency band to be detected. In such case, the diffraction equation does not have an analytic solution. It has been shown [10] that the numerical solution of the diffraction equation for a wideband OA signal can be well described by the analytical solution of the problem for continuous ultrasound using certain wavelength. The width of the spectrum is limited by both the diffraction of optoacoustic signal and transducer transient response. Thus the wavelength corresponding to the frequency of the spectrum maximum can be used considering the long-distant OA beam diffraction. Due to the above-mentioned relationship between the lateral resolution $\Delta y$ and the size of diagnostic window $d$ ("zone of vision" - area of maximum sensitivity of a transducer, around its focus) can be determined.

Obviously, the scattered ultrasonic radiation cannot be detected in full spatial angle. Therefore, the reconstruction of the inhomogeneity distribution has to be performed with an incomplete data set. Consequently, the reconstructed image is expected to be partially distorted [1]. Critical aperture angle $\theta$ in $X Y$ plane is defined by the angle of diffraction divergence of the OA beam. The width of the OA source directivity pattern defines minimal focusing length which allows reconstructing correctly the source.

On the other hand, the aperture angle of the antenna $\varphi$ in the plane $X Z$ is limited by the first critical angle of ultrasound incidence from water to backing.

The resolution in the image plane $\Delta z$ with given aperture angle can be defined from the dependence of the directivity pattern width on the parameter $\varepsilon=\frac{\Delta x}{\Delta z} \leq 1$ [7].

The relationship of the geometric parameters of the antenna (radius of single element curvature $r$, length of antenna $L$, length $l$ and width of single element $2 b_{0}$ ) with the spatial resolutions and the size of the diagnostic window is defined by the geometry of the antenna. It's also necessary to take into account that the capacity of the transducer should exceed well the typical input capacitance of antenna preamplifier. Therefore, the transducer capacity is limited by $C_{\min }=30 \mathrm{pF}$, which means that the radius of curvature of the transducer is also limited.

Besides, at the transition from cylindrical geometry of the piezoelements to the plane system of piezoelements with focusing acoustic lens, the radius of the acoustic lens needs to be calculated [5] and the angular aperture conservation needs to be ensured.

\section{Experimental cases}

Using the calculation algorithm derived for the problem of early breast cancer diagnostics ( $\Delta x=0.1 \mathrm{MM}, d=10 \mathrm{~mm}$ ), parameters of multi-element focused antenna were calculated and the antenna was then developed (Fig. 2).

The focal zone map of a single transducer was plotted using experimental signals obtained by means of the constructed transducer array (Fig. 2$)$. Laser radiation $(\lambda=1.06$ $\mu \mathrm{m}, \tau_{L}=8 \mathrm{~ns}, \nu=500 \mathrm{~Hz}$ ) was delivered by an optical fiber to the ultrasonic generator placed into an immersion liquid. The generator was made of a $20-\mu \mathrm{m}$ thick black polyethylene film with coefficient of absorption $\mu_{a} \cong 1000 \mathrm{~cm}^{-1}$. Laser beam at the surface 
of the polyethylene film was $0.4 \mathrm{~mm}$ that provides the generator as a point source of OA signals. System "optical fiber - generator" could be moved in three dimensions by means of a three-dimensional translator. Scanning area in $X Y$ plane was chosen in a way which allowed completely scanning the entire diagnostic window of the transducer. The obtained frequency band $0.6-10.6 \mathrm{MHz}$ (Fig. 2) corresponds well to the one calculated by the methodology $(1.8-10.8 \mathrm{MHz})$. Also, the focal zone map was numerically calculated using the antenna parameters from the methodology (Fig. 3).
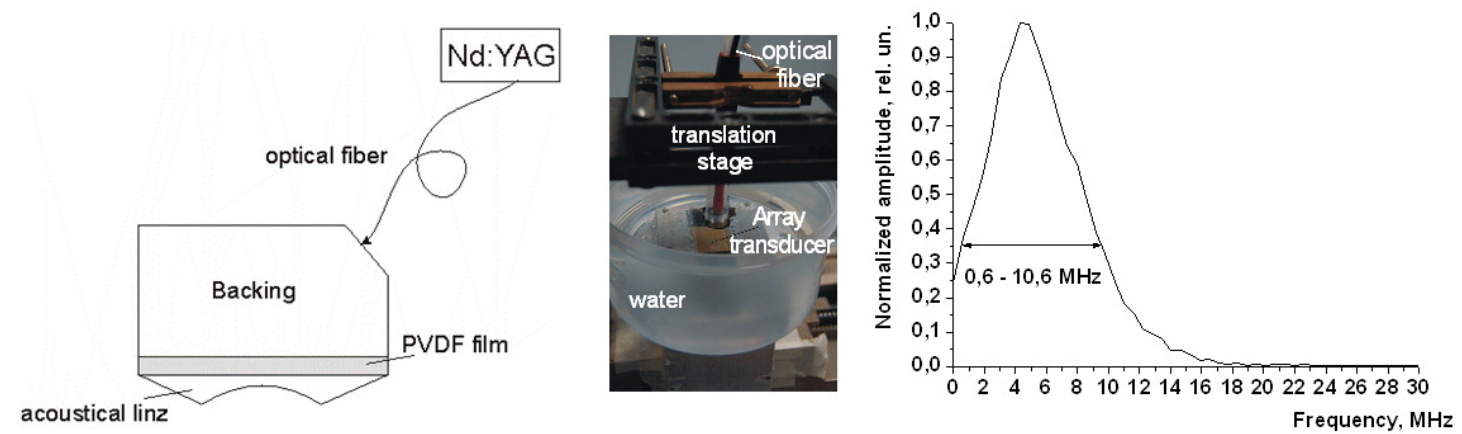

Figure 2. Scheme of the antenna elements disposition (left), experimental setup (center) and spectrum of one of the OA signals obtained by PVDF-transducer (right)

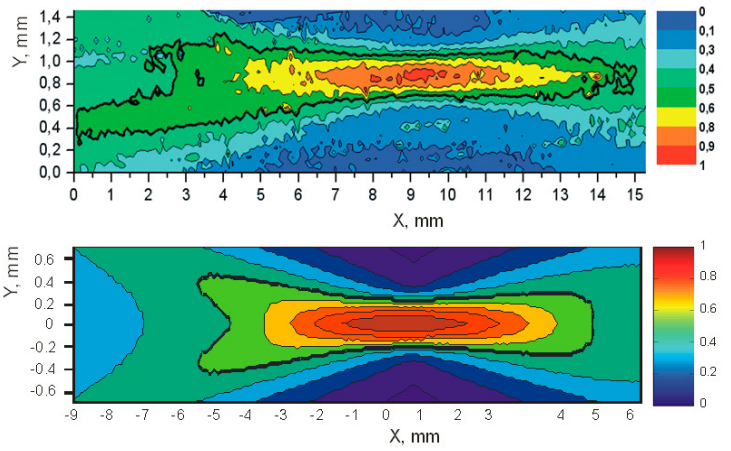

Figure 3. Focal zone maps calculated from the experimentally obtained OA signal (top), numerically simulated using antenna parameters (bottom), black line borders $1 / 2$ level zone, axis $X$ is directed from the transducer

One can see that lateral resolution value and diagnostics window size gained using experimentally obtained and numerically calculated focal zone maps are in a good agreement with the values calculated by the proposed methodology (Table 1)

Table 1. Resolutions $\Delta y, \Delta z$ and diagnostics window size $d$ of 8-element antenna

\begin{tabular}{|c|c|c|c|}
\hline & Experimentally obtained & Numerically calculated & Methodology \\
\hline$\Delta y$ & $0.3 \mathrm{~mm}$ & $0.5 \mathrm{~mm}$ & $0.6 \mathrm{~mm}$ \\
\hline$d$ & $11.3 \mathrm{~mm}$ & $9.9 \mathrm{~mm}$ & $10 \mathrm{~mm}$ \\
\hline
\end{tabular}




\begin{tabular}{|c|c|c|c|}
\hline$\Delta z$ & $2 \mathrm{~mm}$ & $1.6 \mathrm{~mm}$ & $0.3 \mathrm{~mm}$ \\
\hline
\end{tabular}

The point spread function (PSF) was also numerically calculated and experimentally measured for the developed antenna (Fig. 4). The method of the point spread function measurement is described in details in $[6,7,11]$. By means of the point spread function, the lateral resolution $\Delta z$ in the image plane $X Z$ can be determined. As one can see (Table $1)$, image dimensions are in good agreement with their calculated values, but significantly differ to the ones given by the methodology.
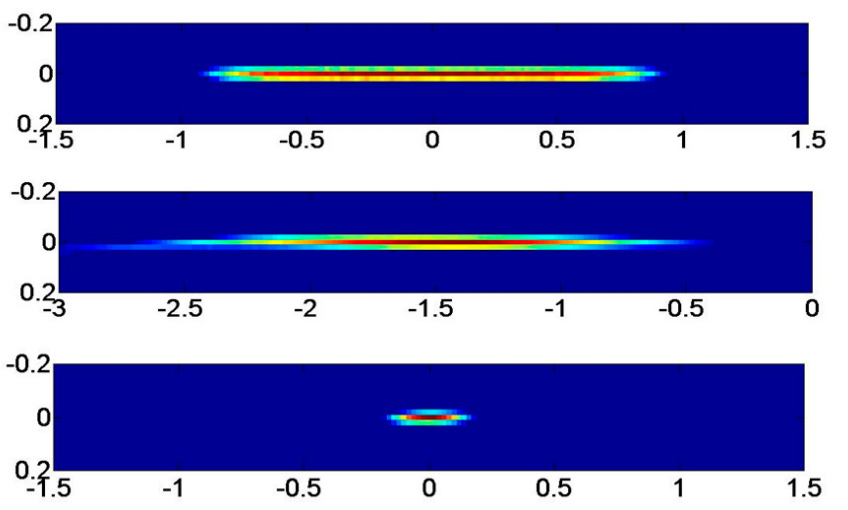

Figure 4. Numerically calculated (top) and experimentally obtained (middle) point spread function of 8elements antenna. Numerically calculated point spread function of 160-elements antenna (bottom)

The reason of so large lateral resolution $\Delta z$ when flare angle is large $\left(\varphi=43^{\circ}\right)$ and antenna is not so long $(L=18 \mathrm{~mm})$ is that transducers are too wide $\left(2 b_{0}=2 \mathrm{~mm}\right)$. Proposed methodology does not account for spatial averaging of wave front by a transducer width, which is considerable for such a small amount of transducers $(N=8)$. Data obtained from 8 transducers is not enough to correctly reconstruct image in that direction (towards the axis $z$ ). It is confirmed by numerical calculation of the point spread function for an antenna with 160 elements and all other parameters unchanged (numerically calculated $\Delta z=0.3 \mathrm{~mm}$, calculated by the methodology $\Delta z=0.3 \mathrm{~mm}$ ).

\section{Conclusions}

The analytical algorithm of main characteristics and geometric parameters calculations of multi-element wideband focused antenna was proposed.

Based on the developed algorithm, the parameters of an 8-element antenna with cylindrical focusing designed for early breast cancer diagnostics with longitudinal resolution in the image plane $\Delta x=0.1 \mathrm{~mm}$ and diagnostics window $d=10 \mathrm{~mm}$ was calculated.

Piezotransducers were made of $110-\mu \mathrm{m}$ thick and 2-mm wide PVDF film and located on a plane with 1-mm step. Focusing was provided by an acoustic lens made of plexiglass, with a flare angle of $57^{\circ}$ and a radius of curvature of $20.6 \mathrm{~mm}$. 
For the developed antenna, focal zone maps were numerically calculated and experimentally measured, and point spread functions were plotted using experimentally obtained and numerically calculated OA signals. Received images confirm the developed algorithm. Discrepancies between experimentally obtained and given values of lateral resolution $\Delta z$ can be explained by the finite width of the transducer and the small number of transducer elements.

\section{Acknowledgements}

This work was supported by Russian Foundation for Basic Research (grant 07-0200940-a) and International Science and Technology Center (grant \#3691).

\section{References}

1. V.E. Gusev, A.A. Karabutov, Laser optoacoustic, M., Nauka, 1991, 278 pp.

2. R.A. Kruger, W.L. Kiser, A.P. Romilly, P. Schmidt, 'Thermoacoustic CT of the breast: Pilot study observations', Proc. SPIE, 4256, pp. 1-5 (2001).

3. V.G. Andreev, A.A. Karabutov, S.V. Solomatin, E.V. Savateeva, V.L. Aleynikov, Y.V. Zhylina, R.D. Fleming, A.A. Oraevsky, 'Opto-acoustic tomography of breast cancer with arc-array transducer', Proc. SPIE, 3916, pp. 36-47 (2003).

4. A.A. Oraevsky, A.A. Karabutov, S.V. Solomatin, E.V. Savateeva, V.G. Andreev, Z. Gatalica, H. Singh, R.D. Fleming, 'Laser optoacoustic imaging of breast cancer in vivo', Proc. SPIE, 2001, vol. 4256, p. 6.

5. A.A. Oraevsky, A.A. Karabutov, 'Ultimate sensitivity of Time-resolved OptoAcoustic detection', Proc. SPIE, 2000, vol. 3916, p. 1.

6. A.N. Zharinov, A.A. Karabutov, V.V. Kozhushko, I.M. Pelivanov, V.S. Solomatin, T.D. Khokhlova, 'Wideband Focused Film Transducer for Optoacoustic Tomography', Acoustical Physics, 2003, vol. 49, no. 6, p. 682.

7. V.V. Kozhushko, T.D. Khokhlova, A.N. Zharinov, I.M. Pelivanov, V.S. Solomatin, A.A. Karabutov, 'Focused array transducer for two-dimensional optoacoustic tomography', J. Acoust. Soc. Am., 2004, vol. 116 (3), pp. 1498-1506.

8. Y. He, Zh. Tang, Zh. Chen, W. Wan, J. Li, 'A novel photoacoustic tomography based on a time-resolved technique and an acoustic lens imaging system', Phys. Med. Biol., 2006, vol. 51, pp. 2671-2680.

9. S. Ermilov, A. Conjusteau, K. Mehta, R. Lacewell, P. M. Henrichs, A.A. Oraevsky, '128-channellaser optoacoustic imaging system (LOIS-128) for breast cancer diagnostics', Proc. SPIE, 2006, vol. 6086, p. 608609-1-12.

10. T.D. Khokhlova, I.M. Pelivanov, A.A. Karabutov, 'Optoacoustic tomography utilizing focused transducers: the resolution study', Appl. Phys. Lett., vol. 92, pp. 024105 1-3 (2008). 
11. A.N. Zharinov, A.A. Karabutov, V.V. Kozhushko, I.M. Pelivanov, V.S. Solomatin, T.D. Khokhlova, 'Spatial Resolution of a Focused Array Transducer for Laser Optoacoustic Tomography', Laser Physics, 2003, vol. 13, no. 11, p. 1.

12. Akhmanov S.A. Nikitin S.Yu., Physical Optics, M., MSU print, 1998, 655 pp. 